\title{
Cloudshine: New Light on Dark Clouds
}

\section{Citation}

Foster, Jonathan B., and Alyssa A. Goodman. 2005. "Cloudshine: New Light on Dark Clouds." The Astrophysical Journal 636 (2): L105-8. https://doi.org/10.1086/500131.

\section{Permanent link}

http://nrs.harvard.edu/urn-3:HUL.InstRepos:41393423

\section{Terms of Use}

This article was downloaded from Harvard University's DASH repository, and is made available under the terms and conditions applicable to Other Posted Material, as set forth at http:// nrs.harvard.edu/urn-3:HUL.InstRepos:dash.current.terms-of-use\#LAA

\section{Share Your Story}

The Harvard community has made this article openly available.

Please share how this access benefits you. Submit a story.

Accessibility 


\title{
CLOUDSHINE: NEW LIGHT ON DARK CLOUDS
}

\author{
Jonathan B. Foster and Alyssa A. Goodman \\ Harvard-Smithsonian Center for Astrophysics, 60 Garden Street, Cambridge, MA 02138 \\ Received 2005 October 22; accepted 2005 November 10; published 2005 December 16
}

\begin{abstract}
We present new deep near-infrared images of dark clouds in the Perseus molecular complex. These images show beautiful extended emission that we model as scattered ambient starlight and name "cloudshine." The brightness and color variation of cloudshine complicates the production of extinction maps, the best tracer of column density in clouds. However, since the profile of reflected light is essentially a function of mass distribution, cloudshine provides a new way to study the structure of dark clouds. Previous work has used optical scattered light to study the density profile of tenuous clouds; extending this technique into the infrared provides a highresolution view into the interiors of very dense clouds, bypassing the complexities of using thermal dust emission, which is biased by grain temperature, or molecular tracers, which have complicated depletion patterns. As new wide-field infrared cameras are used to study star-forming regions at greater depth, cloudshine will be widely observed and should be seen as a new high-resolution tool, rather than an inconvenience.
\end{abstract}

Subject headings: dust, extinction — ISM: structure

\section{INTRODUCTION}

The mass structure of molecular clouds can be studied in a variety of ways, but none are without problems. Molecular line maps can produce high-resolution views, but depletion and excitation variations confound conversion to true column density. Dust emission at submillimeter wavelengths depends on the emitting characteristics of grains, which may vary across molecular clouds. The most direct method for determining the mass structure is extinction mapping, exemplified by NICER (Near-Infrared Color Excess method Revisited; Lombardi \& Alves 2001), which uses the reddening of background stars in the near-infrared to infer the dust column density. The resolution of this method is proportional to the number of background stars visible through the cloud: this is a function of Galactic position, the quantity of obscuring material, and the depth of the images used.

In this Letter we report on near-IR observations originally made to construct an extinction map in the outer regions of the Perseus molecular cloud at moderate column densities $\left(A_{V}<30 \mathrm{mag}\right)$ and relatively high Galactic latitude. In these deep images (complete to $J=20.1, H=20, K_{s}=19.3$ ), there is pronounced emission structure associated with the clouds (see Figs. 1 and 2). This diffuse structure, or "cloudshine," is well explained by scattering of the diffuse interstellar radiation field by dust grains with the optical properties found in Weingartner \& Draine (2001). With deep enough images, it seems likely that every dark cloud becomes a faint reflection nebula in the near-IR, a phenomenon that has been reported a few times before (Lehtinen \& Mattila 1996 and Nakajima et al. 2003) in the context of constraining dust grain models. The structure's ubiquity, relative brightness, and color variation on arcsecond scales complicate the production of extinction maps from deeper exposures where the emission from the extended structure is roughly equal to that of faint background stars. However, the cloudshine also presents a new way to study the mass structure of molecular clouds at subarcsecond resolution.

\section{OBSERVATIONS}

The observations were taken in 2005 January at Calar Alto Observatory on the $3.5 \mathrm{~m}$ telescope, using the new OMEGA
2000 camera. This wide-field near-infrared camera has a $2048 \times 2048$ pixel array and provides a 15'.1 $\times 15$ ' 1 field of view. We covered six science fields and two control fields in three nights of good weather with $\sim 0$ ".6 seeing. We observed with long exposure times at $H$ (45 minutes) and $K_{s}$ (37 minutes) in order to produce the extinction map, and we added short exposures at $J$ ( 6 minutes) to check the reddening law, although these proved useful for studying the diffuse emission. We covered the entirety of B5 with a mosaic of four frames $(J$-band images for two frames were lost due to a pointing error): two dark clouds to the southeast of Perseus, L1448 and L1451, and two control fields outside the boundaries of the cloud. All science fields showed cloudshine, while neither control field did. The control fields were observed with the same exposure times as the science frames and reached the same depths. Additional images and data not in this Letter are available through the COMPLETE (CoOrdinated Molecular Probe Line Extinction and Thermal Emission) Survey Web site. ${ }^{1}$

We did not anticipate extended emission and thus observed without taking off-target sky frames. Instead, we dithered (using a semirandom pattern) around a pointing, obtaining exposures of 2 or $3 \mathrm{~s}$ each. After dark subtraction and division by a flat field, we used the median images from the three images taken before and after a frame to construct a sky. This was carried out by XDIMSUM under IRAF, although the same process was run through ESO's ECLIPSE software to check for consistency. As the extent of the cloudshine was larger than the dither pattern $\left(\sim 30^{\prime \prime}\right)$, our images suffer from self-sky subtraction. We studied the effect of this by creating synthetic data with known shapes, adding on a sky frame derived from data with some random noise and running this sythetic data through the same sky-subtraction routine in XDIMSUM. In general, the effect of self-sky subtraction in such a well-dithered pattern was a small $(<10 \%)$ reduction in surface brightness in a band about the size of the dither pattern around edges of a feature and a dark rim around the edge of the structure. We thus assured ourselves that self-sky subtraction did not substantially alter the morphology of our extended structure. However, future

\footnotetext{
${ }^{1}$ Available at http://cfa-www.harvard.edu/COMPLETE.
} 


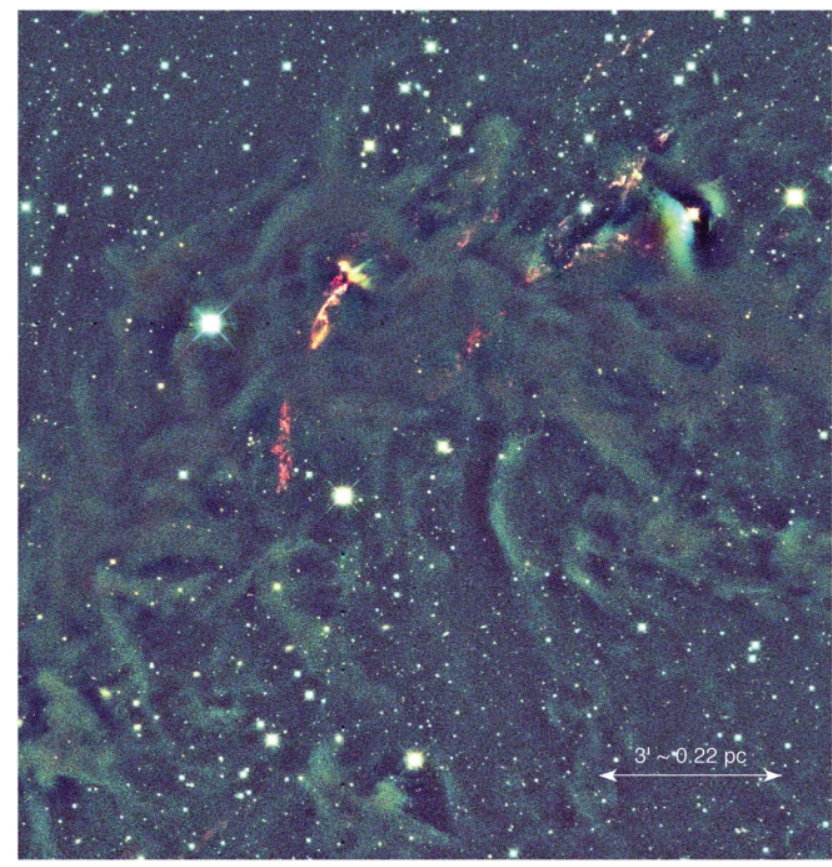

FIG. 1.-L1448 in false color. Component images have been weighted according to their flux in units of $\mathrm{MJy} \mathrm{sr}^{-1} . J$ is blue, $H$ is green, and $K_{s}$ is red. Outflows from young stars glow red, while a small fan-shaped reflection nebula in the upper right is blue-green. Cloudshine, in contrast, is shown here as a muted glow with green edges. Dark features around extended bright objects (such as the reflection nebula) are the result of self-sky subtraction.

observations to study this phenomenon should take off-target frames to obtain a more accurate sky subtraction.

As these data were taken as part of COMPLETE (Goodman 2004; N. Ridge et al. 2005, in preparation) we had immediate access to a variety of other data for the same regions. The diffuse emission traced the location of the $850 \mu \mathrm{m}$ and $1.2 \mathrm{~mm}$ dust continuum emission, and $\mathrm{N}_{2} \mathrm{H}^{+}$and ${ }^{13} \mathrm{CO}$ line emission, so it was clear that cloudshine was associated with the dense portions of the clouds. We made color images to aid in studying the data: Two Micron All Sky Survey (2MASS) stars within the field were used to convert flux per pixel to megajanskys per steradian, and the component images were weighted so that a pixel's color shows the ratio of the fluxes within each band coming from that region of sky. In Figure 1, we have shown L1448 with each component image scaled to the same flux scale in units of $\mathrm{MJy} \mathrm{s} \mathrm{s}^{-1}$, and the region nicely highlights several different physical mechanisms. The outflows are red $\left(K_{s}\right)$, the reflection nebula is blue $(J)$, and the cloudshine is brownish (predominately $H$ and $K_{s}$ ). In Figure 2 we show L1451 with the same weighting of the color components by flux, but with a different optimization of contrast and black point. Additionally, we have overlaid $1.2 \mathrm{~mm}$ continuum dust emission from COMPLETE (M. Tafalla 2006, in preparation) to show how the reddest sections of the cloudshine correspond to the regions of high thermal dust emission and how the edges of the cloudshine trace the contours of the $1.2 \mathrm{~mm}$ emission.

\section{DISCUSSION}

\subsection{Explanations Considered and Rejected}

We considered a variety of possible sources for the cloudshine. L1448 shows a number of bright outflows in the $K_{s}$ image associated with shocked $\mathrm{H}_{2}(v=1-0$ transition); could weaker

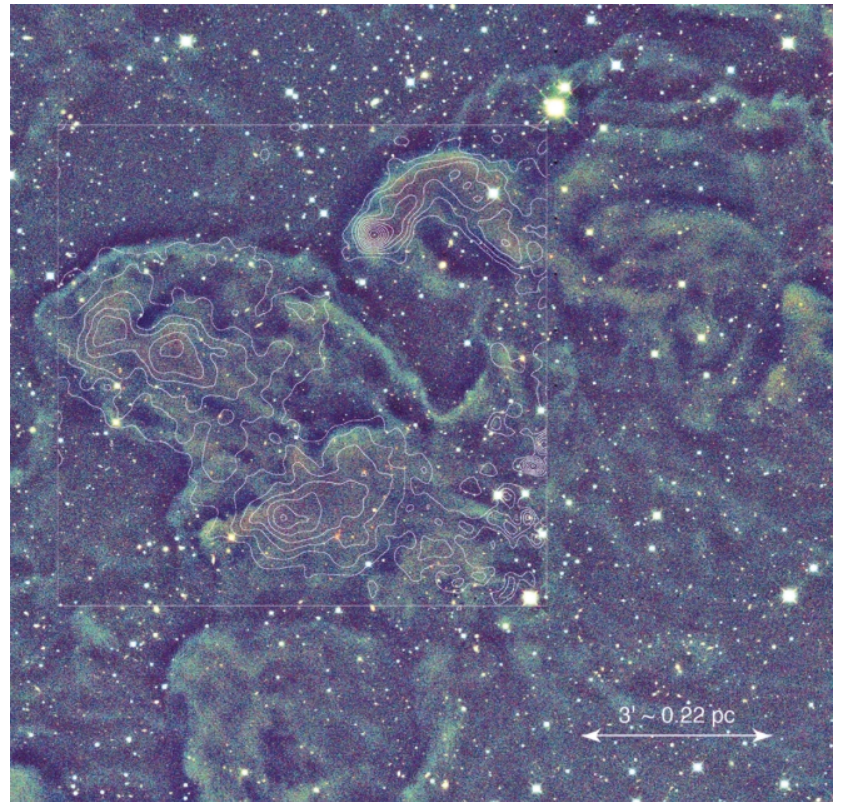

FIG. 2.-L1451 in false color. Again, each component image has been scaled to the same flux scale in units of MJy sr${ }^{-1}$; and $J$ is blue, $H$ is green, and $K_{\text {s }}$ is red. A smaller map of $1.2 \mathrm{~mm}$ dust emission contours from COMPLETE (M. Tafalla 2006, in preparation) has been overlaid, showing that the color of cloudshine is a tracer of density. Redder regions have high dust continuum flux, and the edges of cloudshine match the edges of the dust emission. Dark edges around bright features (particularly noticeable along the northern edges) are the result of self-sky subtraction.

turbulent shocks excite $\mathrm{H}_{2}$ levels in a similar way? Black \& van Dishoeck (1987) present a full spectrum of $\mathrm{H}_{2}$ lines in their Figure 2. The majority of strong lines arise only from UV excitation, which is unlikely in dark clouds. Furthermore, the integrated intensity of these lines is significantly greater in $J$ than in $H$, while our cloudshine is much stronger in $H$ than $J$ (more than can be explained by a few magnitudes of visual extinction between us and the source). Even more unlikely would be thermal emission from shocked grains. To radiate most strongly between $H$ and $K$ requires $1500 \mathrm{~K}$ or shocks traveling on the order of $10 \mathrm{~km} \mathrm{~s}^{-1}$. Stochastic heating of grains (Sellgren 1984) by individual UV photons cannot be ruled out, but the preferential emission from high column density regions indicates that this is not the main mechanism.

New emission features of dust are a possibility. Gordon et al. (2000) report on a strong feature at $1.6 \mu \mathrm{m}$ ( $H$ band) seen in spectra around the reflection nebula NGC 7023. Without spectra of the cloudshine, it is impossible to rule out that such a feature might be contributing to the color of the dust, but this feature is tentatively identified as photoluminescence from amorphous iron disilicide $\left(\beta-\mathrm{FeSi}_{2}\right)$, so, again, the low UV flux in the portions of these dark clouds that give rise to cloudshine makes this effect unlikely to contribute strongly.

\subsection{Modeling the Diffuse Structure as Reflected Starlight}

The simplest explanation is reflected starlight. The appearance of clouds in reflected starlight is a function of the incident radiation field, the optical properties of the grains, and the mass structure of the cloud. This problem simplifies in dark clouds far from stellar clusters since the radiation field is isotropic and a simple function of Galactic latitude. Also, it is difficult to strongly constrain grain models in the near-IR - a relatively 

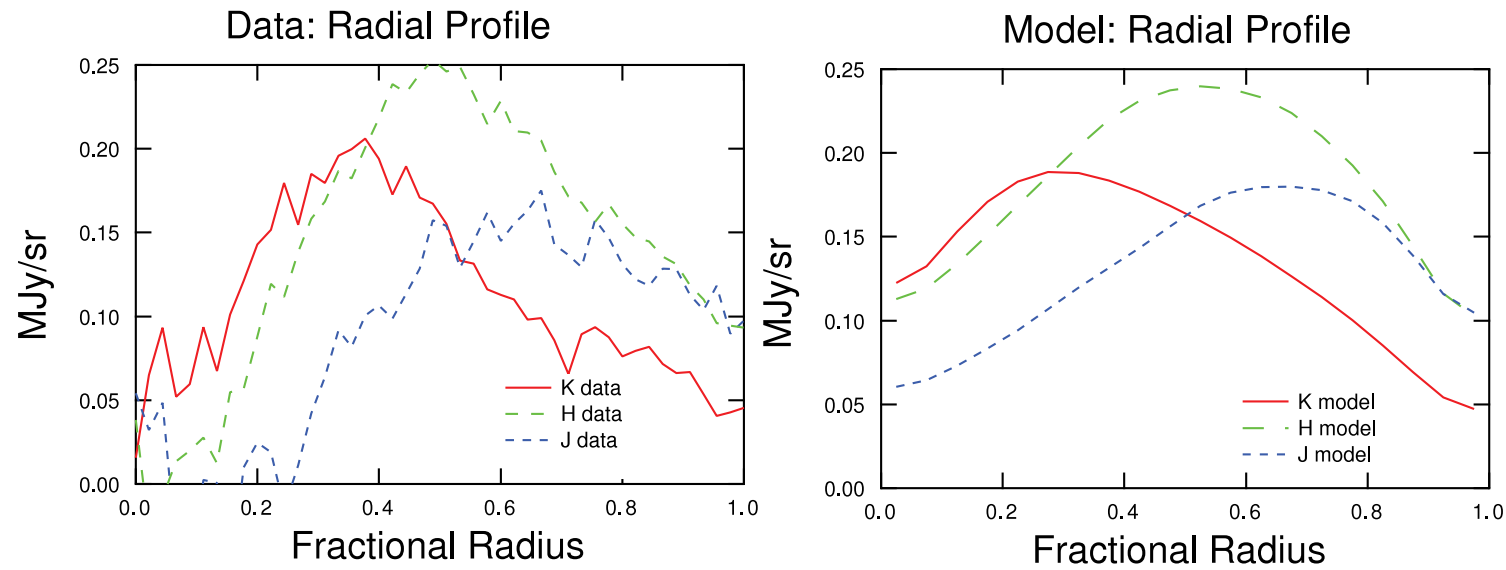

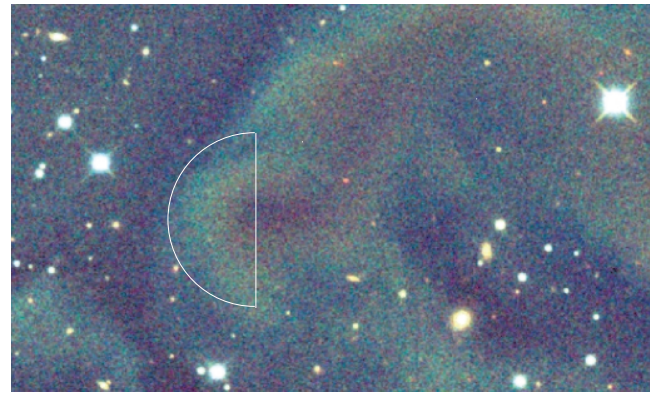

Data Used in Constructing Core Profile

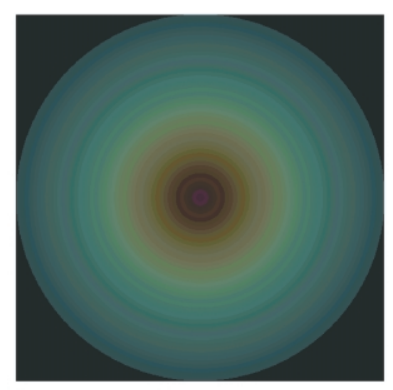

Data Radial Profile

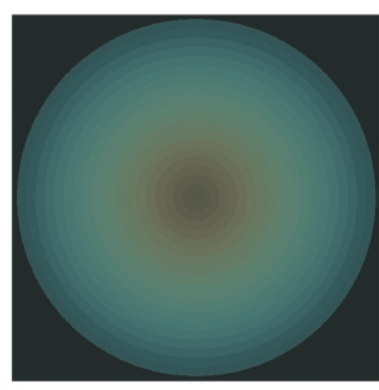

Model Radial Profile

FIG. 3.- Model of cloudshine in one core as reflected interstellar radiation. The lower left panel shows the roughly circular feature we chose to model as a

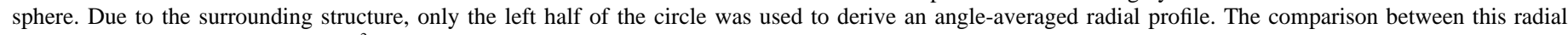

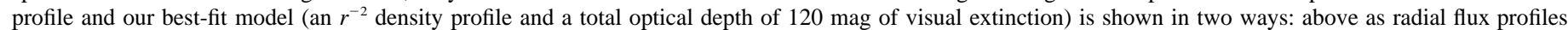

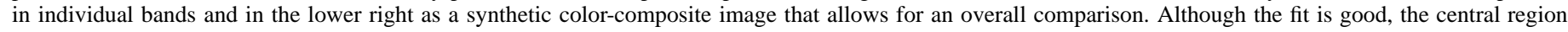

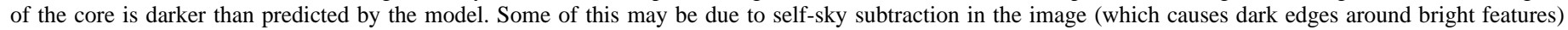
and a nonspherical, nonisotropically illuminated core, and some may be due to a failure to adequately model the density structure at the center of the core.

wide range of parameters produces roughly the same appearance (Lehtinen \& Mattila 1996; Nakajima et al. 2003). Conversely, the exact properties of the dust have little influence on the appearance of scattered light, leaving us with an image that depends mostly on the mass structure of the cloud.

To test the reflection hypothesis, we used a Monte Carlo approach to model the appearance of spherical clouds in the near-infrared. Our study follows the work of Mattila (1970) and Witt \& Stephens (1974) who modeled arbitrary density profiles and dust parameters. As in Witt \& Stephens (1974), a sphere is uniformly illuminated along one axis by a number of photons. These photons enter a cloud consisting of a series of shells of different densities and hence optical depths. The length before scattering is chosen randomly to produce the correct distribution, and if this length exceeds the distance along the current path to the next shell, the photon passes into a new shell, adjusts the remaining optical path length for the new density environment, and continues. When the photon scatters, its weight is reduced by the albedo for the grains, and a new direction is chosen from the Henyey-Greenstein (HG) distribution with a factor $g=\langle\cos \theta\rangle$ characterizing the amount of forward scattering. The HG function is inaccurate at wavelengths far from the optical, where Draine (2003) advocates using a new function, but for the near-IR, the error in the HG function is less than $2 \%$.

Photons eventually escape from the cloud, and their final direction cosines with respect to their initial approach are binned into equal area bins. The problem is then inverted. Since we track each photon along its life history, we weight each entering photon by the weight of the photons in its final bin so that each solid angle bin around the sphere has the same weight of photons leaving through it (in the physical problem this corresponds to isotropic illumination). We then bin the incoming photons by their initial impact parameter, giving the radial profile of the cloud.

By weighting individual shells, we can set up an arbitrary density profile and total optical depth for the cloud. Choosing a certain depth in visual extinction, and adopting the typical ratio of reddening to visual extinction for dark clouds $\left(R_{V}=\right.$ 5.3; Weingartner \& Draine 2001), we obtain optical depths to the center of the cloud for $J, H$, and $K_{s}$ each. We run the simulation three times on the same physical cloud, but with different optical depths, albedos, and values of forward scattering corresponding to the different wavelengths. We must then include the transmitted light that passes through unscattered. This can be calculated analytically for a specified density distribution. This is added to the scattered light profile, and then the illuminating source of light is subtracted out to mimic the effect of sky subtraction.

The final input necessary is the ambient starlight. Lehtinen \& Mattila (1996) model the diffuse Galactic light as a function of Galactic latitude based on $J$ and $K$ data from $C O B E$ (COsmic Background Explorer) on DIRBE (Diffuse InfraRed Background Experiment). They fix the brightness at $H$ so that the colors $(H-K$ and $J-H)$ are the same as the mean color of other spiral galaxies. We adopt their mean surface brightnesses of $I_{J}=0.44, I_{H}=0.61$, and $I_{K}=0.48 \mathrm{MJy} \mathrm{sr}^{-1}$. Since the core we chose to model lies within a larger cloud, we reddened 
this Galactic flux by $5 A_{V}$, the approximate column density of the area as known from COMPLETE extinction maps based on 2MASS (N. Ridge et al. 2005, in preparation).

We modeled the scattered light resulting from power-law density profiles ranging from $r^{-1}$ to $r^{-3}$. The steeper the density profile, the narrower the peak produced in the model, as most radiation is scattered from a small range of optical depth around 1. We then selected one roughly circular dark core in L1451 and made an angle-averaged radial brightness profile. A model with an $r^{-2}$ density profile and a total optical depth of $120 \mathrm{mag}$ of visual extinction reproduces the shape and magnitude of the data radial profiles as shown in Figure 3. This model is a less good fit in the inner portions. It is possible that the linearly spaced shells do a poor job of tracing the density profile at the center, although the physical system must also have a flattened density profile at the center. An exact fit is probably impossible because the core is unlikely to be truly spherical or isotropically illuminated and because self-sky subtraction will somewhat alter the radial profile.

This type of modeling was introduced by Witt et al. (1990), who used reflected optical light to study the structure of dark clouds. Extending this to the infrared has two main advantages. First, deep near-infrared images of dark clouds are likely to be obtained for a number of other reasons (e.g., extinction mapping or studying embedded stars). Second, near-infrared radiation penetrates much deeper into clouds, giving a more complete image of their structure.

\subsection{Studying the Mass Structure of Dark Clouds}

Only in simple cases such as described in the previous section will it be possible to infer the three-dimensional structure of a cloud from cloudshine, but an exciting new work by Padoan et al. (2005) shows that scattered light images may lead directly to a two-dimensional column density map. By illuminating a model cloud, they demonstrate a method by which the color of scattered light can be directly used as an unbiased estimator of the column density. This method raises the possibility of subarcsecond column density maps, a typical linear resolution improvement (in these regions) of 100 times over extinction mapping and 20 times over molecular line or dust emission mapping.

A final application of cloudshine is in comparing models to data. Simulations of turbulent structure produce mass density models of clouds, which can then be illuminated with IR photons, and the resulting image can be statistically compared with our images in a variety of ways (e.g., Principle Component Analysis or the Spectral Correlation Function), to test the validity of the simulations.

\section{CONCLUSION}

As near-infrared cameras and space-born instruments (such as the James Webb Space Telescope) become more efficient, cloudshine will be widely observed in deep exposures. Although it complicates observing strategy on the ground, and makes accurate color photometry of faint sources more difficult, cloudshine is also a useful tool. We have presented here one example of how we may study the density structure of dark clouds where other tools cannot, and we have pointed out how wide-scale near-infrared images of this phenomena could lead to constraints on models of turbulence. Within the fairly large assumptions required, cloudshine can be adequately explained purely as scattered light, although other contributions cannot be firmly ruled out. Isolated, spherical clouds would be the best place to test these models in detail, while larger, more complex clouds provide stronger comparisons with theoretical models.

We thank Elisabeth Adams and Yuri Beletsky for assistance during observing and Ed Churchwell, Bruce Draine, Felice Frankel, Tracy Huard, Phil Myers, Paolo Padoan, Alicia Porras, Ellen Zweibel, and the COMPLETE team (especially João Alves) for comments on this work while it was in progress. We particularly thank Paolo Padoan for providing an advance copy of his numerically oriented companion article to this one. This work was supported by grants from both NASA and the NSF to J. B. F. and A. A. G.

\section{REFERENCES}

Black, J. H.,\& van Dishoeck, E. F. 1987, ApJ, 322, 412

Draine, B. T. 2003, ApJ, 598, 1017

Goodman, A. A. 2004, in ASP Conf. Ser. 323, Star Formation in the Interstellar Medium, ed. D. Johnstone et al. (San Francisco: ASP), 171

Gordon, K. D., et al. 2000, ApJ, 544, 859

Lehtinen, K., \& Mattila, K. 1996, A\&A, 309, 570

Lombardi, M., \& Alves, J. 2001, A\&A, 377, 1023

Mattila, K. 1970, A\&A, 9, 53
Nakajima, Y., Nagata, T., Sato, S., Nagayama, T., Nagashima, C., Kato, D., Kurita, M., \& Kawai, T. 2003, AJ, 125, 1407

Padoan, P., Juvela, M., \& Pelkonen, V.-M. 2005, ApJ, 636, L101

Sellgren, K. 1984, ApJ, 277, 623

Weingartner, J. C., \& Draine, B. T. 2001, ApJ, 548, 296

Witt, A. N., Oliveri, M. V., \& Schild, R. E. 1990, AJ, 99, 888

Witt, A. N., \& Stephens, T. C. 1974, AJ, 79, 948 